\title{
Editorial:
}

\section{La Segregación Escolar Comienza en Educación Infantil}

\section{School segregation Begins in Early Childhood Education}

\author{
F. Javier Murillo ${ }^{1 *}$ \\ Cynthia Duk ${ }^{2}$ \\ ${ }^{1}$ Universidad Autónoma de Madrid, España \\ ${ }^{2}$ Universidad Central de Chile, Chile
}

No cabe duda alguna: la asistencia de los niños y niñas a Educación Infantil tiene una clara influencia en su desarrollo posterior. Efectivamente, podemos llamar a este nivel, Educación Infantil, Educación Inicial, Educación Preescolar, Educación Parvularia, Educación de la Primera Infancia, usando la Clasificación Internacional de la Educación CINE, al margen de cómo se la denomine en la actualidad contamos con sólidas evidencias científicas de que los niños y niñas que han tenido acceso tempranamente a la educación tienen un mejor desarrollo integral y que los beneficios de esta escolarización siguen dejando su impronta muchos años después.

En esa lógica, todos los países del mundo han redoblado esfuerzos para incrementar la tasa de ingreso a la educación inicial y los fondos invertidos en esta etapa han subido de forma importante en los últimos años. Sin embargo, el hecho de que en muchos lugares esta etapa no sea obligatoria, ni gratuita, ha llevado a que se convierta en un factor más de inequidad de los sistemas educativos.

De esta forma, se escolarizan en el nivel inicial en mayor medida niños y niñas de familias con mayores recursos sociales, económicos y culturales, con lo que el posible efecto compensador de la Educación Infantil se pervierte, convirtiéndose en un factor que profundiza estas incipientes inequidades. Pero es que, además, la segregación escolar en esta etapa es incluso mayor que en las posteriores y sus efectos, si cabe, más devastadores (Fram y Kim, 2012; Hogrebe et al, 202 1; Piazza y Frankenberg, 2019). Veámoslo con un mínimo de detalle desde diversas perspectivas.

De entrada, hay que asumir que tenemos muy pocos estudios que estimen la magnitud de la segregación escolar en Educación Infantil, y menos trabajos aún que la comparen con lo que acaece en otras etapas educativas. La necesidad de grandes bases de datos con muestras estadísticamente representativas supone una seria limitación. No obstante, afortunadamente, contamos con trabajos muy recientes que abordan el problema. Un estudio del Urban Institute (2019), con datos de los Estados Unidos, muestra que la segregación en este nivel es mucho más alta que en Primaria e incluso mayor para los niños y niñas de 0 a 3 años que para los de 3 a 5 años. En Iberoamérica destacan dos estudios igualmente recientes que indagan en este tema, encontrando resultados similares. El primero de ellos realizado en Uruguay (Ferrando et al., 2020) estudia la segregación en Educación Inicial, Primaria, Secundaria y Técnica en ese país. Los hallazgos muestran que la segregación más alta se da en Educación Infantil y que, como en el resto de las etapas, está aumentando en forma ininterrumpida en los últimos años. Estos resultados

*Contacto: javier.murillo@uam.es 
son análogos a los encontrados por la Cátedra Unesco en Educación para la Justicia Social (2021) en un estudio llevado a cabo en una ciudad española de tamaño medio. Se trata solo de tres estudios, pero muestran ya una clara tendencia.

Esta alta segregación tiene graves consecuencias a corto, medio y largo plazo. Para empezar es importante recordar que los beneficios de una exposición temprana a la diversidad son múltiples. Así, a los seis meses de edad, los niños y niñas ya han comenzado a hacer distinciones étnico-raciales, y, en algún momento entre las edades de 3 y 5 , es posible observar la aparición de prejuicios raciales (Hirschfeld, 2008). También tenemos evidencias (p. ej., Howes y Wu, 1990) de que los niños que asistieron a centros o programas de educación infantil con alta diversidad en Primaria generan más interacciones y amistades con niños de diferentes culturas y grupos étnico/raciales. Experiencias que, sin duda, ayudan a prevenir o reducir el desarrollo de prejuicios y estereotipos, contribuyendo así a la creación de una sociedad más cohesionada (Zhou et al., 2019). En este sentido, el desarrollo de actitudes de solidaridad, respeto, empatía y comprensión hacia los demás, se consigue creando entornos educativos inclusivos en la primera infancia, que proporcionen oportunidades de aprender a convivir con la diversidad desde la más temprana edad.

Por otra parte, los centros, jardines infantiles y programas de Educación Infantil con una mayor proporción de niños y niñas de familias de bajos recursos, con un menor capital cultural, de origen extranjero o de grupos culturales minoritarios tienen menos probabilidades de ser considerados de alta calidad, porque carecen de los recursos tangibles necesarios para el aprendizaje (Reid et al., 2015). A lo que se añade que en muchos países, los y las educadoras de estos programas y centros suelen tener una menor formación y experiencia. Incluso, algún estudio ha encontrado que los niños y niñas que asisten a Educación Infantil en aulas segregadas, ya sea según nivel socioeconómico, origen étnico o cultural tienen un peor desarrollo del lenguaje que aquellos que asisten a centros más diversos (Reid, 2016; Schecter y Bye, 2007).

Un último aspecto hace referencia a la formación y a la actitud de los educadores y educadoras responsables de la educación en esta etapa. En muchas ocasiones, muestran prejuicios implícitos que los llevan a esperar comportamientos desafiantes de los niños de familias vulnerables, de grupos étnicos-culturales minoritarios o de origen extranjero, y demuestran expectativas más bajas hacia ellos. No hace falta insistir en la fuerza del efecto Pigmalión para convertir esos prejuicios en hechos. Curiosamente, en numerosos países la formación requerida para proporcionar una atención integral a los niños y niñas de corta edad es menor que en etapas posteriores, por lo que en general los y las educadoras perciben que no tienen una preparación adecuada para trabajar con la diversidad del alumnado. Podemos llamarlo paradojas de la vida, o una forma de invertir menos, incluso en los salarios que suelen ser los más bajos, minusvalorando una etapa tan crucial para el desarrollo personal y social o más que cualquier otra.

Además, la segregación escolar en Educación Infantil es la puerta de entrada a la segregación en Educación Primaria y, con ello, en Educación Secundaria. Efectivamente, en gran parte de los países existe continuidad entre los niveles y centros educativos de Educación Infantil y Primaria, de tal forma que hay poco cambio de escuela. Si consideramos que la educación infantil no es siempre gratuita, ya en esos momentos se produce una selección temprana de familias con más recursos en centros privados, selección que suele mantenerse posteriormente. 
Con estos escasos párrafos creemos haber demostrado sobradamente la importancia de prestar una mayor atención a la segregación escolar en Educación Infantil. Hasta ahora ha sido un tema prácticamente desconocido y, con ello, evitado. Cierto es que aún no tenemos suficientes estudios que estimen su magnitud, y los pocos planes contra la segregación existentes apenas la consideran como una etapa prioritaria para abordar. Pero no hay alternativa, o atacamos la segregación en los primeros años de la trayectoria educativa, o se corre peligro de que acabe siendo crónica en la escolaridad y en la sociedad.

\section{Referencias}

Cátedra UNESCO en Educación para la Justicia Social. (2021). Diagnóstico a la Segregación escolar en Getafe. Cátedra UNESCO en Educación para la Justicia Social.

Ferrando, F., Hernández-Almeida, M., Oreiro, C., Seijas, M. N. y Urraburu, J. (2020). Evolución de la segregación socioeconómica en la educación pública de Uruguay. REICE. Revista Iberoamericana sobre Calidad, Eficacia y Cambio en Educación, 18(4), 143-169. https://doi.org/10.15366/reice2020.18.4.006

Fram, M. S. y Kim, J. (2012). Segregated from the Start: Peer Context in Center-based Child Care, Children ES Schools, 34(2), 71-82. https://doi.org/10.1093/cs/cds011

Hirschfeld, L. (2008). Children's developing conceptions of race. En S. Quintana y C. McKown (Eds.) Handbook of race, racism, and the developing child (pp. 37-54). John Wiley \& Sons.

Hogrebe, N., Pomykaj, A. y Schulder, S. (2021). Segregation in Early Childhood Education and Care in Germany: Insights on Regional Distribution Patterns Using National Educational Studies. Discourse: Journal of Childhood \& Adolescense Research/Diskurs Kindheits-und Jugendforschung, 16(1), 36-56. https://doi.org/10.3224/diskurs.v16i1.04

Howes, C. y Wu, F. (1990). Peer interactions and friendships in an ethnically diverse school setting. Child Development, 61(2), 537-541. https://doi.org/10.2307/1131113

Piazza, P. y Frankenberg, E. (2019). Segregation at an early age. Center for Education and Civil Rights, Penn State University.

https://cecr.ed.psu.edu/sites/default/files/Segregation_At_An_Early_Age_Piazza_Fran kenberg_2019.pdf

Reid, J. L. (2016). Racial/ethnic diversity and language development in the preschool classroom. En E. Frankenberg, L. M. Garces y M. Hopkins (Eds.), School integration matters: Researchbased strategies to advance equity (pp. 39-55). Teachers College Press.

Reid, J. L., Kagan, S. L., Hilton, M. y Potter, H. (2015). A better start: Why classroom diversity matters in early education. The Poverty \& Race Research Action Council (PRRAC). http://www.prrac.org/pdf/A_Better_Start.pdf

Schecter, C. y Bye, B. (2007). Preliminary evidence for the impact of mixed-income preschools on low-income children's language growth. Early Childhood Research Quarterly 22(1), 137-146. https://doi.org/10.1016/j.ecresq.2006.11.005

Urban Institute. (2019). Segregated from the start: Comparing segregation in early childhood and K-12 education. Urban Institute. https://www.urban.org/features/segregated-start

Zhou, S., Page-Gould, E., Aron, A., Moyer, A. y Hewstone, M. (2019). The extended contact hypothesis: A meta-analysis on 20 years of research. Personality and Social Psychology Review, 23(2), 132-160. https://doi.org/10.1177/1088868318762647 